\title{
Integrando electricidad, ondas y calor en la enseñanza de la física antes y durante el confinamiento por COVID-19
}

\author{
Integrating electricity, waves, and heat in physics teaching before and \\ during confinement by COVID-19
}

\begin{abstract}
Roberto Bernardo Usca Veloz. ${ }^{1}$, Juan Carlos Muyulema Allaica. ${ }^{2}$, \& Grace Alexandra Velasteguí Bósquez. ${ }^{3}$
\end{abstract}

\begin{abstract}
.
Introduction. Education during the COVID-19 health contingency has had to migrate to the online environment. This change has impacted not only educational institutions and their staff, but also families. Objective. This article aims to compare the academic performance of university students, integrating the subject of electricity, waves and heat, for the teaching of physics, before and during the confinement by COVID-19. Methodology. To achieve the objective, a study was developed based on the positivist paradigm, which has the particularity of observing, measuring, describing, using the hypothetical-deductive method, in a non-experimental descriptive type of work, based on precise data derived from academic results. For this purpose, the case of the subject of electricity, waves and heat was analyzed within the career of Pedagogy of Mathematics and Physics of the State University of Bolivar (UEB). The universe was made up of all 4 th semester students taking the subject. The target research population consisted of $19+$

\footnotetext{
${ }^{1}$ Universidad Estatal de Bolívar (UEB), Facultad de Ciencias de la Educación, Sociales, Filosóficas y Humanísticas. Guaranda, Ecuador. email: rusca@ueb.edu.ec ORCID ID: https://orcid.org/0000-00016600-052X

${ }^{2}$ Universidad Internacional SEK (UISEK), Facultad de Ingeniería y Ciencias Aplicadas, Posgrado. Quito, Ecuador. email: juan.muyulema@uisek.edu.ec ORCID ID: https://orcid.org/0000-0002-9663-8935

${ }^{3}$ Unidad Educativa Galo Plaza Lasso, Responsable de la asignatura de matemáticas. Echeandía, Ecuador. email: gracealexandravelastegui@gmail.com ORCID ID: https://orcid.org/0000-0001-6391-9733
} 
$15=34$ students, whose academic results were available for the academic periods September 2019 - February 2020 and June - September 2020, before and during the COVID-19 confinement, respectively. Results. The analyzed results show analogies in academic performance, thus sustaining that there are other factors affecting students, which show that the pandemic situation highlighted the socio-educational inequality, since education has remained dependent on virtuality, where technological resources and internet access are indispensable. Conclusions. Virtual education processes must be generated incorporating modern teaching-learning dynamics, in addition to compressing the digital divide and socio-educational inequality.

Keywords: teaching, physics, academic performance, pandemic, sustainability.

\section{Resumen.}

Introducción. La educación durante la contingencia sanitaria por COVID-19 ha tenido que migrar al entorno en línea. Este cambio ha impactado no sólo a las instituciones educativas y su personal, sino también a las familias. Objetivo. A través del presente artículo se pretende comparar el rendimiento académico en estudiantes universitarios, integrando la asignatura de electricidad, ondas y calor, para la enseñanza de la física, antes y durante el confinamiento por COVID-19. Metodología. Para lograr el objetivo, se desarrolló un estudio basado en el paradigma positivista, el cual tiene por particularidad observar, medir, describir, empleándose el método hipotético - deductivo, en un trabajo de tipo descriptivo no experimental, a partir de unos datos precisos derivados de los resultados académicos. Para ello se analizó el caso de la asignatura de electricidad, ondas y calor dentro de la carrera de Pedagogía de las Matemáticas y la Física de la Universidad Estatal de Bolívar (UEB). El universo lo conformaron todos los estudiantes de $4^{\circ}$ semestre que tomaron la asignatura. La población objetivo de investigación estuvo compuesta por $19+15=34$ estudiantes, de los que se ha tenido acceso a sus resultados académicos, en los períodos académicos Septiembre 2019 - Febrero 2020 y Junio - Septiembre 2020, antes y durante el confinamiento por COVID-19 respectivamente. Resultados. Los resultados analizados demuestran analogías en el rendimiento académico, sosteniendo de esta forma que existen otros factores de afectación en los estudiantes, que dan cuenta de que la situación de pandemia puso en evidencia la desigualdad socioeducativa, ya que la educación ha subsistido dependiente a la virtualidad, donde los recursos tecnológicos y el acceso a internet son indispensables. Conclusión. Se deben generar procesos de educación virtual incorporando modernas dinámicas de enseñanza aprendizaje, a más de comprimir la brecha digital y desigualdad socioeducativa.

Palabras claves: enseñanza, física, rendimiento académico, pandemia, sostenibilidad.

\section{Introducción.}

La sociedad del tercer milenio (1 de enero de 2001 al 31 de diciembre del año 3000) presenta exigencias y posibilidades diversas, se caracteriza por permutaciones precipitadas en el campo de la Ciencia, Tecnología, Sociedad y Ambiente (CTSA) 
(Lamarre \& Hernández, 2020). En la actualidad se han desarrollado numerosos trabajos investigativos, como lo hacen Camacho, Valenzuela, \& Caldera (2017); Olvera, et al., (2018); Niño \& Fernández (2019); Gil (2019); Sánchez, Herrera, \& Rodríguez (2020); Abeleira \& Vázquez (2021), acentuando resultados sobre la forma de enseñar y aprender ciencias y en especial de la Física Básica en la Universidad, y este a su vez contrasta con las demandas de la sociedad actual, las cuales apuntan a la necesidad que el área de ciencias pueda proporcionar el desarrollo de competencias y habilidades cognitivas en los estudiantes. Sumado a esto, la implementación de estrategias pedagógicas y didácticas basadas en la incorporación de nuevas tecnologías. De esta forma, el papel del docente universitario coexistirá como mediador del conocimiento, no el centro del proceso educativo y así permitir al alumno desarrollar habilidades conceptuales e incrementar su motivación por la investigación y el desarrollo de un pensamiento racional para progresar en el conocimiento de la realidad objetiva.

Estos nuevos formatos demandan enérgicamente de docentes formados y empoderados para que alcancen a tomar decisiones pedagógicas en el aula, sobre la base de los lineamientos curriculares determinados y las circunstancias y situaciones de sus estudiantes (Lamarre \& Hernández, 2020). Si bien durante la contingencia sanitaria por Coronavirus (COVID-19), un sinnúmero actores educativos (docentes, estudiantes) se han visto incitados a colocar a disposición materiales y recursos educativos en disímiles plataformas, el cuerpo docente precisa tiempo y orientación para conocerlos, explorarlos y contar con criterios elementales para la toma de decisiones en relación a su uso (Urzúa, Rodríguez, Martínez, \& Eustaquio, 2020). Estimaciones han alertado que la pandemia ha inducido la mayor paralización de la historia en los regímenes educativos, que ha afectado aproximadamente a 1.600 millones de alumnos, situados en cerca de 190 países en todos los continentes. Los cierres de escuelas y otros centros educativos de todos los niveles del sistema han afectado significativamente al $94 \%$ de los alumnos y alumnas de todo el mundo, una cifra que remonta al $99 \%$ en países de ingreso bajo y mediano bajo (UNESCO, 2020).

Desde el punto de vista pedagógico, educación en la virtualidad conjetura el riesgo de detrimento del vínculo presencial y puede sin lugar a duda generar tensiones por la sobreexposición de los docentes y estudiantes (Sánchez, Herrera, \& Rodríguez, 2020), o por los conflictos para conservar la relación y la mediación pedagógica en el proceso de enseñanza (Vélez-Loor, Vallejo-Valdivieso, \& Moya-Martínez, 2020).

Desde el punto de vista social, el acrecentamiento del desempleo formal y la pobreza, sumado a mayores horizontes de violencia doméstica y de dificultades de salud física y mental, conduces a que todo el personal educativo se vea enfrentado a los conflictos y tensiones que experimentan las familias, sin contar, en numerosos casos, con los recursos materiales o profesionales ineludibles para abordarlas (Urzúa, Rodríguez, Martínez, \& Eustaquio, 2020). Este escenario forja un desgaste emocional, agobio y estrés entre los actores educativos (Urzúa, Rodríguez, Martínez, \& Eustaquio, 2020).

La educación para todos y todas es un derecho humano universal, que debe ser asegurado en todo momento y lugar, independiente del contexto y situaciones, incluyendo los 
escenarios de emergencias (Pérez-López, Vázquez, \& Cambero, 2021). Los escenarios de emergencias son entendidos como desastres que destruyen, por un período de tiempo, las condiciones usuales de la vida, por lo tanto, trastornan, obstaculizan o retardan la realización del derecho a la educación (Serrano-Castro, et al., 2019).

No obstante, asegurar la continuidad educativa no es simple y está colmado de circunspectos desafíos (Jiménez \& Ruiz, 2021). Pese a que existen recomendaciones en investigaciones recientes, el tema se registra como "abierto" y remotamente carece de evidencia concluyente que consiga orientar todas las acciones (Montenegro, 2020). Un sin número de países afectados del mundo y en especial en la región latinoamericana están exponiendo e implementando respuestas, pero el panorama de las políticas públicas se describe como emergente y inteligible, la evidencia científica y documentación es por si decirlo "gris" y, a menudo, escasea de referencias, lo que hace dificultoso establecer lo que funciona y lo que no funciona en esta etapa aguda de emergencia sanitaria (Expósito \& Marsollier, 2020; Jiménez \& Ruiz, 2021).

La continuidad educativa en Ecuador establece un fenómeno emergente, mientras que se modula como una extensión clave del derecho humano universal a la educación en situación de emergencia (Peñaherrera-Acurio, Peñaherrera-Acurio, \& Espinoza-Beltrán, 2021). Lo presentado parece indicar que es una temática tan relevante que es imposible de omitir, pero la evidencia es tan limitada que es difícil de asumir.

Por lo cual hoy en día cobra relevancia indagar y conocer la mirada de los estudiantes universitarios de ciencias respecto a su percepción del proceso de aprendizaje antes y durante la contingencia sanitaria por COVID-19, particularmente en asignaturas de carácter práctico, cuyo lugar natural de trabajo es el laboratorio. A razón de esto, el objetivo de esta investigación es comparar el rendimiento académico en estudiantes universitarios, integrando la asignatura de electricidad, ondas y calor, para la enseñanza de la física, antes y durante el confinamiento por COVID-19.

Así, la investigación se desarrolló en función del paradigma positivista, el cual tiene por particularidad observar, medir, describir, empleándose el método hipotético - deductivo, desde el punto de vista metodológico se apoyó en un trabajo de tipo descriptivo no experimental. Para ello se analiza el caso de la asignatura de electricidad, ondas y calor dentro de la carrera de Pedagogía de las Matemáticas y la Física de la Universidad Estatal de Bolívar (UEB) en los niveles de enseñanza superior ( $4^{\circ}$ semestre), en el período académico Septiembre 2019 - Febrero 2020 (Ex-ante COVID-19) y, por otro lado, en el período académico Junio - Septiembre 2020 (Durante COVID-19).

\section{Metodología.}

Este trabajo investigativo se realizó dentro de una amplia actividad investigadora de la Facultad de Ciencias de la Educación, Sociales, Filosofía y Humanísticas de la UEB donde se abordan la influencia y claves del sistema educativo en la sociedad contemporánea, concretamente en Ecuador, eje clave en las actuales políticas educativas, por supuesto, pedagógicas en el país. La tarea se centró en compilar datos rigurosos sobre 
datos precisos derivados de los resultados académicos, resultantes de la enseña de la asignatura electricidad, ondas y calor dentro de la carrera de Pedagogía de las Matemáticas y la Física - UEB, temas que habitualmente son objeto de polémica dado al fracaso del aprendizaje, relacionado enérgicamente con factores externos de la propia enseñanza, como son: la falta de capacidad intelectual del estudiantado, su falta de interés, trabajo deficiente, bajo nivel de preparación anterior, entre otros.

El proceso investigativo se desarrolló en función del paradigma positivista, el cual asume por particularidad observar, medir, y describir, se requirió la aplicación de un método hipotético - deductivo, metodológicamente se apoyó en un trabajo de tipo descriptivo no experimental, puesto que se pretende investigar las implicaciones del hecho diferencial antes y durante el confinamiento por COVID-19, a partir de datos precisos procedentes de los resultados académicos. Para ello se analiza el caso de la asignatura de electricidad, ondas y calor dentro de la carrera de Pedagogía de las Matemáticas y la Física - UEB en los niveles de enseñanza superior ( $4^{\circ}$ semestre). El universo de estudio lo conformaron todos los estudiantes de $4^{\circ}$ semestre que tomaron la materia. La población objetivo de investigación se centró en el análisis de 19+15=34 estudiantes, de los que se tuvo acceso a los documentos que contienen sus resultados académicos, en el período académico Septiembre 2019 - Febrero 2020 (Ex-ante COVID-19) y, por otro lado, en el período académico Junio - Septiembre 2020 (Durante COVID-19). Se trata de dos grupos de alumnos, que atendiendo al período se distribuyen como se expone en la Tabla 1.

Tabla 1. Distribución de la muestra

\begin{tabular}{cccc}
\hline Estrato & & Frecuencia & Porcentaje \\
\hline \multirow{2}{*}{ Grupo A (Ex-ante COVID-19) } & Hombres & 13 & $38 \%$ \\
\cline { 2 - 4 } & Mujeres & 6 & $18 \%$ \\
\hline Subtotal A & & 19 & $56 \%$ \\
\hline \multirow{2}{*}{ Grupo B (Durante COVID-19) } & Hombres & 8 & $24 \%$ \\
\cline { 2 - 4 } & Mujeres & 7 & $21 \%$ \\
\hline Subtotal B & & 15 & $44 \%$ \\
\hline Total & & $\mathbf{3 4}$ & $\mathbf{1 0 0 \%}$ \\
\hline \multirow{2}{*}{ Estudiantes absentistas } & Hombres & 1 & \\
\cline { 2 - 4 } & Mujeres & 2 & \\
\hline
\end{tabular}

Fuente: Los autores basados en la nómina de estudiantes en el período académico Septiembre 2019 - Febrero 2020 (Ex-ante COVID-19) y Junio - Septiembre 2020 (Durante COVID-19).

En la Tabla 1, se observa que el número total de estudiantes a ser examinados ascendió a 34, 13 mujeres y 21 hombres, lo que proporciona muestras estadísticamente equivalentes, aunque reducidas. Del mismo modo, se ha considerado el problema existente del absentismo y abandono de los estudios universitarios que podría condicionar de alguna manera las estadísticas de los resultados obtenidos, 1 alumna y 2 alumnos dentro de esta categoría. Por lo tanto, en las comparativas estadísticas realizadas no se han analizado los casos de estudiantes que habitualmente, y por causas ajenas a la coyuntura académica, no participaron con normalidad en las actividades académicas planificadas, excluyéndolos en el tratamiento de datos. Por otro lado, obsérvese que la escala de evaluación cuantitativa grado y posgrado en la UEB, esta oscila entre 0 puntos como resultado mínimo, y un máximo de 10 puntos, siendo habitual el mínimo suficiente de 6.49 puntos 
para el progreso y promoción entre nivel y semestre. Con relación a las particularidades sobre fiabilidad y validez del estudio, se debe exteriorizar que los datos han sido extraídos de los expedientes personales y que estos documentos reflejan unos resultados derivados de procedimientos rigurosos de cuantificación de rendimiento académico. Por otro lado, toda esta información fue tratada con elevados niveles de seguridad, precisión y confidencialidad.

\section{Resultados.}

\section{Aprendizaje de la Física Básica en Universitarios}

La enseñanza de las ciencias experimentales ha ido ligada a una transmisión de los conocimientos teóricos por parte del docente a sus alumnos y con escasa participación práctica (Esteves, Chenet, Pibaque, \& Chávez, 2020). Esta metodología tradicional, que potencia lo memorístico por encima de otras competencias, si no es acompañada con otros métodos, resulta poco eficiente en el proceso de enseñanza-aprendizaje (Romero, Stoessel, \& Rocha, 2020). Se considera que para un mayor rendimiento del aprendizaje se debe aplicar una metodología que debe asemejarse al método científico, que se basa en la investigación (Prieto, 2020).

El trabajo experimental en Física, asignatura de la formación básica en carreras de grado con especialidad en Matemáticas y Física, debería aportar al desarrollo de los aprendizajes vinculados con el aprendizaje de las ciencias básicas, así como tecnologías básicas y aplicadas (este aspecto abarca por un lado, la inclusión de las actividades experimentales en el plan de estudios, considerando la carga horaria mínima; y por el otro, la disponibilidad de infraestructura y equipamiento) (Vivanco, 2020). Así, la Carrera de Pedagogía de las Matemáticas y la Física de la UEB, en su malla curricular se oferta la asignatura de Electricidad, Ondas y Calor, y dentro de sus contenidos se abordan temas concernientes a Oscilaciones y Ondas. Normalmente las prácticas de laboratorio se realizan de manera presencial en el laboratorio de Física de la Carrera, pero por la emergencia sanitaria provocada por COVID-19 que vive el país se tuvo que cambiar de metodología de enseñanza a la educación virtual.

Ante el contexto de emergencia sanitaria por COVID-19, algunos autores mencionan que la educación virtual abre brechas hacia la desigualdad social ya que no está al alcance para todos los estudiantes debido a motivos económicos, sociales, culturales, entre otros, sin embargo, en este contexto actual de pandemia se hace necesario el tratar de garantizar el proceso educativo en al menos una cierta parte de la población, como refiere Esteves, et al., (2020); González-Zamar, Abad-Segura, \& Bernal-Bravo (2021) se debe pensar en estrategias de enseñanza-aprendizaje pero ahora en forma virtual, con el fin de atenuar estas desigualdades y reducir el impacto que está teniendo la educación en todo el mundo. Es así que los educadores tienen la posibilidad de utilizar la analítica del aprendizaje como una importante herramienta de conocimiento, puesto que esta estrategia debería ser propicia para comprender el progreso de los estudiantes, reconocer conceptos problemáticos e identificar a los estudiantes con dificultades. 
Por lo cual hoy en día cobra relevancia indagar y conocer la mirada de los estudiantes universitarios de ciencias respecto a su percepción del proceso de aprendizaje durante la contingencia sanitaria por COVID-19, particularmente en asignaturas de carácter práctico, cuyo lugar natural de trabajo es el laboratorio. Así, nos planteamos como hipótesis de investigación: Los resultados de rendimiento académico en estudiantes universitarios, integrando la asignatura de electricidad, ondas y calor, para la enseñanza de la física, son diferentes antes y durante el confinamiento por COVID-19.

\section{Comparación de los resultados de rendimiento académico}

En tabla 2 aparecen reflejados los resultados académicos obtenidos, en el período académico Septiembre 2019 - Febrero 2020 (Ex-ante COVID-19) y, por otro lado, en el período académico Junio - Septiembre 2020 (Durante COVID-19).

Tabla 2. Resultados del rendimiento académico Ex-ante y durante la COVID-19

\begin{tabular}{cccccccc}
\hline $\begin{array}{c}\text { Escala } \\
\text { cualitativa }\end{array}$ & $\begin{array}{c}\text { Escala } \\
\text { cuantitativa } \\
\text { grado y } \\
\text { posgrado }\end{array}$ & Equivalencias & $\begin{array}{c}\text { Valoración } \\
\text { de } \\
\text { asignatura, } \\
\text { curso, } \\
\text { módulo o su } \\
\text { equivalente }\end{array}$ & $\begin{array}{c}\text { Ex-ante } \\
\text { COVID- } \\
\mathbf{1 9}\end{array}$ & $\begin{array}{c}\text { \% } \\
\text { Durante } \\
\text { COVID- } \\
\mathbf{1 9}\end{array}$ & $\%$ \\
\hline A & $9-10.00$ & Excelente & Aprueba & 11 & $58 \%$ & 8 & $53 \%$ \\
\hline B & $8-8.49$ & Muy bueno & Aprueba & 6 & $32 \%$ & 2 & $13 \%$ \\
\hline C & $7-7.49$ & Bueno & Aprueba & 2 & $11 \%$ & 3 & $20 \%$ \\
\hline D & $6-6.49$ & Regular & Recuperación & 0 & $0 \%$ & 2 & $13 \%$ \\
\hline E & Menos de 6 & Deficiente & No aprueba & 0 & $0 \%$ & 0 & $0 \%$ \\
\hline & & Total & & $\mathbf{1 9}$ & $\mathbf{1 0 0 \%}$ & $\mathbf{1 5}$ & $\mathbf{1 0 0 \%}$ \\
\hline
\end{tabular}

Fuente: Los autores basados en los resultados académicos obtenidos, en el período académico Septiembre 2019 - Febrero 2020 (Ex-ante COVID-19) y Junio - Septiembre 2020 (Durante COVID-19).

Los resultados de rendimiento académico (Informe parcial de aprendizaje) conformadas por una muestra de 19 estudiantes en el grupo de control (Ex-ante COVID-19) y 15 experimental (Durante COVID-19), perteneciente al cuarto semestre de la carrera de Pedagogía de las Matemáticas y la Física de la Universidad Estatal de Bolívar, revelaron para el grupo de control que 11 estudiantes (58\%) alcanzan los aprendizajes con un valoración de "Excelente", es decir se ubican en la escala cuantitativa de 9,00 a 10.00, mientas tanto 6 estudiantes (32\%) alcanzan a la equivalencia de "Muy bueno" en tal razón se ubican en la escala cuantitativa de 8,00 a 8,49 , en tanto 2 estudiantes (11\%) se hallan en la equivalencia de "Bueno" y se encuentran dentro de la escala cuantitativa de 7 a 7.49 , no se registran datos correspondientes para las equivalencias de "Regular" y "Malo". Por otro lado para el caso del grupo experimental, 8 estudiantes (53\%) alcanzan los aprendizajes con un valoración de "Excelente", situándose en la escala cuantitativa de 9,00 a 10.00 , en tanto 3 estudiantes (20\%) se hallan en la equivalencia de "Bueno" y se encuentran dentro de la escala cuantitativa de 7 a 7.49, seguidamente 2 estudiantes (13\%) alcanzan a la equivalencia de "Muy bueno" en tal razón se ubican en la escala cuantitativa de 8,00 a 8,49, en similar porcentaje 2 estudiantes (13\%) se hallan en la equivalencia de "Regular" y se encuentran dentro de la escala cuantitativa de 6 a 6.49, no se registran 
datos correspondientes para la equivalencia "Deficiente", tal como se exterioriza en la Figura 1.

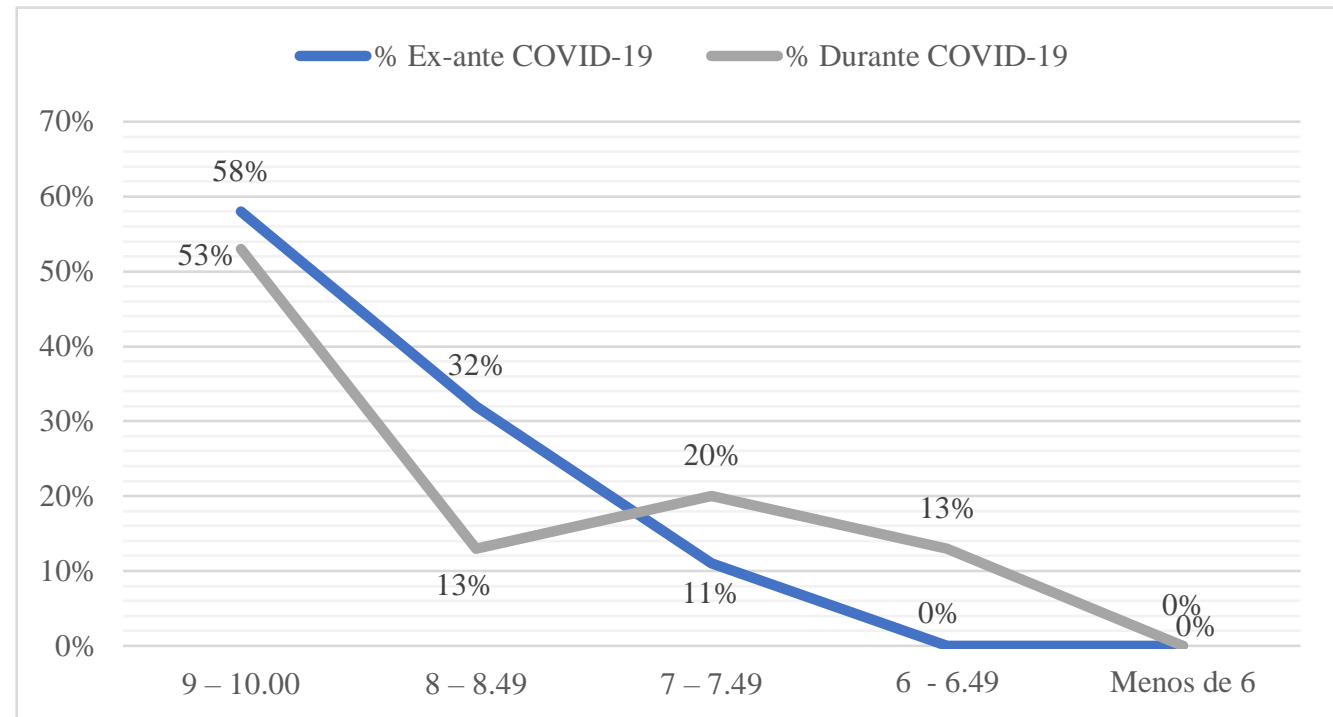

Figura 1. Resultados de rendimiento académico Ex-ante y durante la COVID-19

Fuente: Tabla 2: Resultados del rendimiento académico Ex-ante y durante la COVID-19

\section{Examinación de las diferencias de los resultados de rendimiento académico}

La t-Student, primeramente, se diseñó para explorar las diferencias entre dos muestras independientes y pequeñas $(\mathrm{N}<30)$ que tengan distribución normal y homogeneidad en sus varianzas. Sánchez (2015), hace constancia en la normalidad de las dos muestras como perentorio en el desarrollo de la prueba.

La metodología de la t-Student, sigue los siguientes pasos:

1. Ensayar que cada una de las muestras posee una distribución normal;

2. Conseguir para cada una de las muestras:

a) el tamaño necesario de las muestras a explorar $\left(\mathrm{n}_{1}\right.$ y $\left.\mathrm{n}_{2}\right)$,

b) sus concernientes medias $\left(\mathrm{m}_{1} \mathrm{y} \mathrm{m}_{2}\right)$,

c) sus varianzas dadas $\left(\mathrm{v}_{1} \mathrm{y} \mathrm{v}_{2}\right)$;

3. Probar la homogeneidad de varianza (homocedasticidad);

4. En el caso de que exista homogeneidad en esas varianzas:

a) instaurar la diferencia entre las medias: $\mathrm{m} 1-\mathrm{m} 2$,

b) calcular la varianza común de las dos muestras estudiadas.

$$
\boldsymbol{v} \boldsymbol{c}=\frac{\left(\left(n_{1}-1\right) v_{2}+\left(n_{2}-1\right) v_{2}\right)}{\left(n_{1}+n_{2}-2\right)}
$$

Es decir, la varianza común (vc) es equivalente a un promedio de las varianzas de las dos muestras estudiadas en el cual los pesos para ese promedio son similares al tamaño, menos uno ( $\mathrm{n}-1)$ en cada una de las muestras, 
c) con el dato de la varianza común de las dos muestras estudiadas, se calcula el error estándar de la diferencia de las medias ESM.

$$
E S M=\sqrt[2]{(v c) \frac{n_{1}+n_{2}}{\left(n_{1}\right)\left(n_{2}\right)}}
$$

5. Posteriormente, la t-Student es igual al cociente de la diferencia de medias entre el ESM calculado anteriormente.

6. Finalmente, en concordancia con nuestra hipótesis nula y alterna se procede a demostrar que existe diferencia entre las medias de las muestras estudiadas, se coteja con una tabla de t-Student con grado de libertad igual a $n_{1}+n_{2-2}$ y se deduce el valor de $p$.

Paso 1: prueba de normalidad de cada una de las muestras.

En el grafico 2 se observa los resultados obtenidos de la prueba estadística de AndersonDarling, mediante el software Minitab 19. El valor $p \leq \alpha=0.05$, indica un riesgo de 5\%, por lo tanto, se puede concluir que para el caso Ex-ante COVID-19 $(p=0.009)$ sus datos no siguen una distribución normal, y para el caso Durante COVID-19 ( $p=0.08)$ se logra concluir que los datos alcanzan una distribución normal, tal como se exterioriza en la Figura 2.

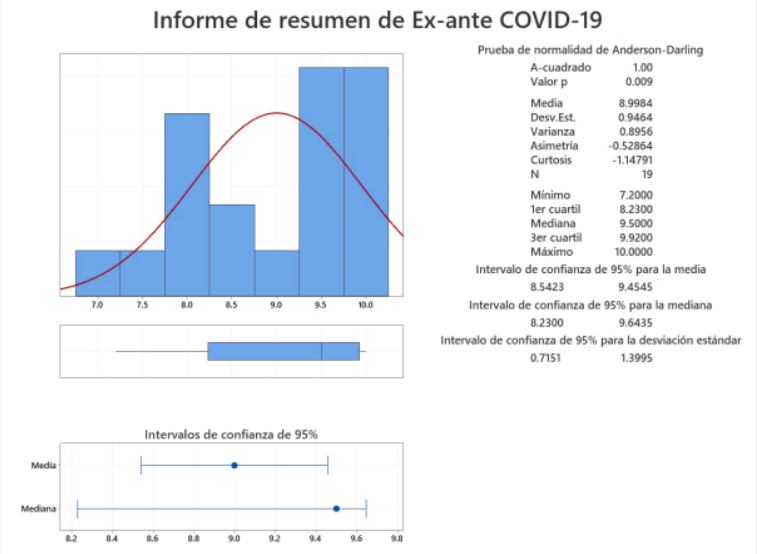

(a)

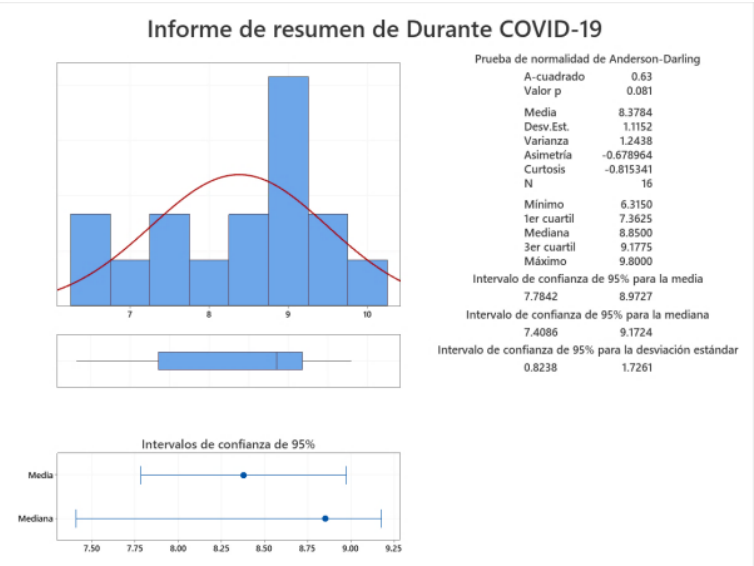

(b)

Figura 2. Prueba de normalidad de las muestras Ex-ante y durante la COVID-19 Fuente: Elaboración propia a partir de datos del rendimiento académico Ex-ante y durante la COVID19

Paso 2: prueba t-test aun estando consciente que una de las muestras (Ex-ante COVID19) no posee normalidad.

Paso 3: prueba para la homogeneidad de varianzas; se logra considerar que son homogéneas, puesto que la $p=0.620$.

Paso 4: Para este apartado, seguimos tres caminos, que son:

(i) establecimiento de la diferencia de medias $=0.028$ 
(ii) cálculo ve a las muestras.

$$
\begin{gathered}
\boldsymbol{v} \boldsymbol{c}=\frac{\left(\left(n_{1}-1\right) v_{2}+\left(n_{2}-1\right) v_{2}\right)}{\left(n_{1}+n_{2}-2\right)} \\
\boldsymbol{v} \boldsymbol{c}=\frac{(18 \times 0.8956)+(14 \times 1.2438)}{19+15-2} \\
\boldsymbol{v} \boldsymbol{c}=\frac{15.0408+17.4132}{32} \\
\boldsymbol{v} \boldsymbol{c}=1.014
\end{gathered}
$$

(iii) cálculo de error estándar de las diferencias de las medias

$$
\begin{gathered}
E S M=\sqrt[2]{(v c) \frac{n_{1}+n_{2}}{\left(n_{1}\right)\left(n_{2}\right)}} \\
E S M=\sqrt[2]{(1.014) \frac{34}{285}} \\
E S M=\sqrt[2]{0.121} \\
E S M=0.347
\end{gathered}
$$

Paso 5: el valor de la t-test será:

$$
\begin{gathered}
t=\frac{\text { Diferencia de las medias }}{E S M} \\
t=\frac{8.998-8.378}{0.347} \\
t=1.786
\end{gathered}
$$

Paso 6: hipótesis:

Ho: Los resultados de rendimiento académico en estudiantes universitarios, integrando la asignatura de electricidad, ondas y calor, para la enseñanza de la física, son iguales antes y durante el confinamiento por COVID-19.

H1: Los resultados de rendimiento académico en estudiantes universitarios, integrando la asignatura de electricidad, ondas y calor, para la enseñanza de la física, son diferentes antes y durante el confinamiento por COVID-19.

Los grados de libertad (GL), para cotejar con la tabla de t-Student son $19+15-2=32$, consultando el valor de $p$ es 0.090 .

Por lo tanto, el valor $p>0,05$, no podemos concluir que existe una diferencia significativa. En tanto, no existe diferencia entre los resultados de rendimiento académico en estudiantes universitarios, integrando la asignatura de electricidad, ondas y calor, para la enseñanza de la física, antes y durante el confinamiento por COVID-19. 


\section{Discusión}

La enseñanza de las ciencias básicas, específicamente, las asignatura que relacionan a la Física, debe generarse en ambientes con interconectividad de plataformas que contribuyan de forma significativa al fomento de un aprendizaje integrador y dinámico, promoviéndose sistemáticamente la vinculación de lo teórico con la cotidianidad que envuelve al estudiante, de esa forma, el saber se establece como un eje esencial para una adecuada formación de una persona reflexiva de corte competitivo (Olvera, Pérez, Méndez, \& Ramírez, 2018). Sin embargo, enseñar bajo esta modalidad en lo referente a asignaturas de ciencias básicas, es un reto, puesto que en estos escenarios se experimenta un proceso que genera incertidumbre, pero además un aprendizaje que establecerá nuevos procesos pedagógicos a seguir para lograr un mejor rendimiento académico (Esteves, Chenet, Pibaque, \& Chávez, 2020). Bajo este contexto se puede evidenciar desde la práctica docente universitaria, que el mayor de los retos es lograr en los estudiantes una motivación genuina, para ello se les debe brindar experiencias de aprendizaje que contengan estrategias y metodologías de enseñanza con la ayuda de las TIC, en sintonía con esto, la enseñanza de la Física, con referencia con el laboratorio de Física se le puede llevar a cabo con la ayuda de simuladores, videos, entre otros.

Reconociendo eficazmente la importancia que las herramientas tecnológicas asumen para dar respuesta a las dificultades que enfrenta el ser humano y para el adelanto y la mejora de la sociedad, este trabajo investigativo se planteó con el objetivo principal de comparar el rendimiento académico en estudiantes universitarios, integrando la asignatura de electricidad, ondas y calor, para la enseñanza de la física, antes y durante el confinamiento por COVID-19. A la vista de los descubrimientos detectados por el estudio, el proceso de adaptación a la virtualidad no se está originando en las mejores condiciones, al menos en lo que respecta al alumnado universitario. De acuerdo con este argumento, y basado en la experiencia docente se puede decir que, si bien el claustro de profesores está haciendo uso de una modalidad de enseñanza síncrona, se transita sobre unas nuevas necesidades, afectadas indudablemente por una sobrecarga de trabajo y la falta de una adecuada planificación y gestión, estas nuevas realidades están dificultando en gran medida su proceso de aprendizaje. Adicionalmente, a lo dicho Montenegro (2020) expone el impacto que están encontrando los estudiantes para comunicarse con sus compañeros, lo intentan disminuir con las aplicaciones con las que están familiarizados, como WhatsApp. Sin embargo, tomado en cuenta los resultados de la investigación y conjeturando sus problemas aun no podemos concluir que existe una diferencia significativa entre los resultados de rendimiento académico en estudiantes universitarios, integrando la asignatura de electricidad, ondas y calor, para la enseñanza de la física, antes y durante el confinamiento por COVID-19.

En lo que respecta al uso programas de videoconferencia como Zoom, Google Meet, Jitsi y Teams, no parecen venir a dar respuesta a las necesidades de un sinnúmero actores educativos (docentes, estudiantes), no obstante se lo considera como un recurso útil y valioso para el aprendizaje virtual sincrónico (Roig-Vila, Urrea-Solano, \& MermaMolina, 2021), su manejo les genera innegables dificultades, sobre todo como resultado 
de la falta de conocimientos, problemas de conexión y la remisión entre la imagen y el sonido (Expósito \& Marsollier, 2020); restricciones que igualmente han sido puestas de manifiesto con el uso de distintas aplicaciones con similares tipologías (Rocha, 2020). En definitiva, la educación bajo el escenario de confinamiento se ha valido de entornos virtuales sincrónicos y asincrónicos de aprendizaje. Este entorno ha venido a ahondar la desigualdad socioeducativa y la brecha digital, puesto que la educación ha quedado dependiente a la virtualidad, en donde los recursos tecnológicos y el acceso a Internet son indispensables. A ello se suma la educación de posgrados y el ámbito de la instrucción no formal.

Por otra parte, el prepararse, a corto, mediano y largo plazo, para comprimir la brecha digital pone en partida sinergias virtuosas de inclusión social y cultural para los estudiantes universitarios, generando oportunidades para enfrentar futuras crisis (González-Zamar, Abad-Segura, \& Bernal-Bravo, 2021). Más allá de las habilidades y acciones educativas agrupadas al aprendizaje, es muy distinguido formar para el autocuidado y el desarrollo efectivo de destrezas para la gestión de riesgos para la educación virtual, así como informar a los estudiantes sobre los tipos de protección como usuarios habituales de Internet, especialmente tomando en cuenta las actuales situaciones de incremento del tiempo de conexión (Usca-Veloz, et al., 2019; Esteves, et al., 2020; Jiménez \& Ruiz, 2021). No obstante, se debe considerar que, no todos los actores educativos están preparados bajo la misma condición en correspondencia con los conocimientos, competencias, actitudes, aptitudes y aprendizajes concretos requeridos para desarrollar y poner en práctica estrategias de autocuidado ante los nuevos escenarios pedagógicos que les permitan aprovechar las oportunidades que ofrece Internet y reducir los riesgos o a su vez saber enfrentarlos.

Finalmente, la acción pedagógica y las nuevas demandas se ponen de cara con el personal docente con una falta de conocimientos tecnológicos, problemas de conexión y una disponibilidad de recursos que tienden a ser innegablemente insuficientes para los retos que supone adecuar la oferta educativa y los formatos pedagógicos a estudiantes en entornos menos favorecidos. De la experiencia docente universitaria, se puede argumentar que, ya antes de la pandemia el personal docente de la región contaba con escasas oportunidades de formación continua para la introducción o para el trabajo con alumnado en escenarios menos favorecidos y de mayor diversidad. Conjuntamente, las nuevas circunstancias han solicitado que el profesorado manipule plataformas $y$ metodologías virtuales con las que no precisamente se encontraba acostumbrado. Sin embargo, la necesidad de ajuste a las condiciones actuales de educación y de formación sincrónica o asincrónica se ha traducido, igualmente, en un compendio de responsabilidades y exigencias que aumentan persuasivamente el tiempo de trabajo que las y los docentes demandan para preparar recursos y materiales para las clases, asegurar conexiones conformes y hacer seguimiento al estudiantado en formatos diversos. 


\section{Conclusiones}

- En el marco de la suspensión de las actividades académicas presenciales, la necesidad de conservar la continuidad de los aprendizajes ha impuesto serios desafíos que los países de la región y en especial el Ecuador han abordado por medio de diferentes opciones y soluciones en relación con los calendarios académicos y las formas de ejecución del currículo, por medios no presenciales. Para realizar los ajustes se requiere tomar en cuenta las particularidades de los currículos nacionales, los recursos disponibles, la capacidad para generar procesos de educación virtual sincrónica o asincrónica, a más de comprimir la brecha digital y desigualdad socioeducativa del país.

- En lo que corresponde a los resultados de rendimiento académico (Informe parcial de aprendizaje) conformadas por una muestra de 19 estudiantes en el grupo de control (Ex-ante COVID-19) y 15 experimental (Durante COVID-19), perteneciente al cuarto semestre de la carrera de Pedagogía de las Matemáticas y la Física de la Universidad Estatal de Bolívar UEB, revelaron que particularmente, en asignaturas de carácter práctico como la materia de electricidad, ondas y calor, cuyo lugar natural de trabajo es el laboratorio, no existe una diferencia significativa entre los resultados de rendimiento académico en estudiantes universitarios, antes y durante el confinamiento por COVID-19. Bajo estas derivaciones obtenidas se confirman las primeras aproximaciones del estudio, sosteniendo que existen otros factores que pueden afectar al buen desempeño de los estudiantes universitarios, que dan cuenta de que el escenario de pandemia puso en evidencia la desigualdad socioeducativa y la brecha digital entre instituciones por un lado las públicas y por el otro las privadas, superponiéndose quienes tuvieron mejor acceso a los recursos tecnológicos y a Internet, puesto que la educación ha quedado dependiente a la virtualidad, en donde los recursos tecnológicos y el acceso a Internet son indispensables.

\section{Referencias bibliográficas.}

Abeleira, O. J., \& Vázquez, V. N. (2021). Examineof real physical systems with the aid of video analysis favorsexperimental research performance. RITI Journal, 9(18), 1-13. doi:https://doi.org/10.36825/RITI.09.18.001

Camacho, R. A., Valenzuela, G. V., \& Caldera, F. M. (2017). Modelización de una actividad de la física para mejorar la enseñanza del concepto de función. IE Revista de investigación educativa de la REDIECH, 8(15), 57-67.

Esteves, F. Z., Chenet, Z. M., Pibaque, P. M., \& Chávez, R. M. (2020). Estilos de aprendizaje para la superdotación en el talento humano de estudiantes universitarios. Revista de Ciencias Sociales, XXVI(2), 225-235. 
Expósito, C. D., \& Marsollier, R. G. (2020). Virtualidad y educación en tiempos de COVID-19. Un estudio empíricoen Argentina. Educación y Humanismo, 22(39), 1-22. doi:https://doi.org/10.17081/eduhum.22.39.4214

Gil, V. V. (2019). Objetos virtuales de aprendizaje en la enseñanza de la física: un caso de aplicación. Revista Internacional de Aprendizaje en Ciencia, Matemáticas y Tecnología, 5(1), 9-17. doi:https://doi.org/10.37467/gka-revedumat.v5.1856

González-Zamar, M. D., Abad-Segura, E., \& Bernal-Bravo, C. (2021). COVID-19 and creative university learning spaces. Research trends. International Journal of Educational Research and Innovation (IJERI), 15(1), 82-100. doi:https://doi.org/10.46661/ijeri.5126

Jiménez, G. Y., \& Ruiz, G. M. (2021). Reflexiones sobre los desafíos que enfrenta la educación superior en tiempos de COVID-19. Economía y Desarrollo, 165(Supl.1 ) , e3.

Lamarre, V., \& Hernández, S. A. (2020). Cultivando física y matemática: ciencias exactas y naturales aplicadas al desarrollo de una huerta agroecológica y comunitaria. Revista de Enseñanza de la Física, 32(2), 69-77.

Montenegro, D. D. (2020). Comunicación grupal en Whatsapp para el aprendizaje colaborativo en la coyuntura COVID-19. Hamut'ay, 7(2), 34-45. doi:http://dx.doi.org/10.21503/hamu.v7i2.2131

Niño, V. J., \& Fernández, M. F. (2019). Una mirada a la enseñanza de conceptos científicos y tecnológicos a través del material didáctico utilizado. Espacios, 40(15), e4.

Olvera, A. M., Pérez, T. L., Méndez, S. A., \& Ramírez, D. M. (2018). Interacción entre físicos y profesoras de preescolar para desarrollar estándares de ciencia. RIDE. Revista Iberoamericana para la Investigación y el Desarrollo Educativo, 9(17), 741-768. doi:https://doi.org/10.23913/ride.v9i17.404

Peñaherrera-Acurio, W. P., Peñaherrera-Acurio, S. J., \& Espinoza-Beltrán, P. S. (2021). Covid- 19: La transformación de la educación en el Ecuador mediante la inclusión de herramientas tecnológicas en las clases virtuales. Dominio de las Ciencias, 7(1), 837-848.

Pérez-López, E., Vázquez, A. A., \& Cambero, R. (2021). Educación a distancia en tiempos de COVID-19: Análisis desde la perspectiva de los estudiantes universitarios. RIED. Revista Iberoamericana de Educación a Distancia,, 24(1), 331-350. doi:http://dx.doi.org/10.5944/ried.24.1.27855

Prieto, A. J. (2020). Una revisión sistemática sobre gamificación, motivación y aprendizaje en universitarios. Revista Interuniversitaria, 32(1), 73-99. doi:http://dx.doi.org/10.14201/teri.20625 
Rocha, E. J. (2020). Active methodologies, the key to changing schools and their application intimes ofpandemic. INNOVA Research Journal, 5(3.2), 33-46. doi:https://doi.org/10.33890/innova.v5.n3.2.2020.1514

Roig-Vila, R., Urrea-Solano, M., \& Merma-Molina, G. (2021). La comunicación en el aula universitaria en el contexto del COVID-19 a partir de la videoconferencia con Google Meet. RIED. Revista Iberoamericana de Educación a Distancia, 24(1), 197-220. doi:http://dx.doi.org/10.5944/ried.24.1.27519

Romero, R. E., Stoessel, A. F., \& Rocha, A. (2020). Un estudio de diseño sobre la implementación de laboratorios remotos en la enseñanza de la física universitaria: la observación del trabajo de los estudiantes. Revista de enseñanza de la física, 32(1), 75-91.

Sánchez, I. R., Herrera, E. D., \& Rodríguez, C. E. (2020). Eficacia de resolución colaborativa de problemas en el desarrollo de habilidades cognitivo lingüísticas y en el rendimiento académico en física. Formación universitaria, 13(6), 191-204. doi:http://dx.doi.org/10.4067/S0718-50062020000600191

Sánchez, T. R. (2015). t-Student. Usos y abusos. Revista mexicana de cardiología, 26(1), $59-61$.

Serrano-Castro, A. K., Muyulema-Allaica, J. C., Usca-Veloz, R. B., \& González-Rivera, M. M. (2019). Aplicación de la metodología STAR para el análisis del riesgo sísmico de la provincia Bolívar - Ecuador. Ciencia Digital, 3(4), 227-249. doi:https://doi.org/10.33262/cienciadigital.v3i4.968

UNESCO. (2020). La educación en tiemposde la pandemia de COVID-19. Santiago: CEPAL, OREALC/UNESCO. Obtenido de https://repositorio.cepal.org/bitstream/handle/11362/45904/1/S2000510_es.pdf

Urzúa, M. D., Rodríguez, D. P., Martínez, V. M., \& Eustaquio, R. R. (2020). Aprender ciencias experimentales mediante TIC en tiempos de covid-19: percepción del estudiantado. Praxis \& Saber, 11(27), e11447. doi:https://doi.org/10.19053/22160159.v11.n27.2020.11447

Usca-Veloz, R. B., Muyulema-Allaica, J. C., Espinosa-Ruiz, C. G., Sánchez-Macías, R. A., Velasteguí-Bósquez, G. A., \& Caspi-Pilamunga, W. M. (2019). La interconexión digital de objetos habituales con internet y sus aplicaciones para la empresa y la Industria 4.0: Review. Revista Internacional de Investigación e Innovación Tecnológica - RIIIT. 7(41), 1-19.

Vélez-Loor, M. D., Vallejo-Valdivieso, P. A., \& Moya-Martínez, M. E. (2020). Recursos didácticos virtuales en proyectos de ciencias naturales en período de confinamiento por COVID-19. Epísteme Koinonía, 3(5), e183. doi:https://doi.org/10.35381/e.k.v3i5.760 
Vivanco, A. A. (2020). Teleducación en tiempos de COVID: brechas de desigualdad. CienciAmérica, 9(2), 1-10. doi:http://dx.doi.org/10.33210/ca.v9i2.307

\section{Ciencia}




\section{PARA CITAR EL ARTÍCULO INDEXADO.}

Usca Veloz, R. B., Muyulema Allaica, J. C., \& Velasteguí Bósquez, G. A. (2021). Integrando electricidad, ondas y calor en la enseñanza de la física antes y durante el confinamiento por COVID-19 . ConcienciaDigital, 4(1.2), 405-421. https://doi.org/10.33262/concienciadigital.v4i1.2.1607

\section{LCiencia}

El artículo que se publica es de exclusiva responsabilidad de los autores y no necesariamente reflejan el pensamiento de la Revista Conciencia Digital.

El artículo queda en propiedad de la revista y, por tanto, su publicación parcial y/o total en otro medio tiene que ser autorizado por el director de la Revista Conciencia Digital.

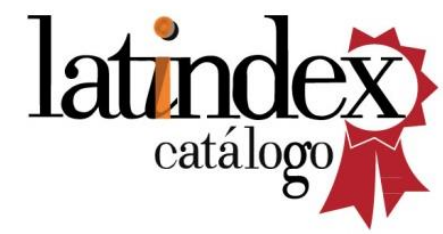

